\title{
Enunciación
}

\section{El aula agonal: una mirada a las relaciones discursivas entre profesor y estudiantes desde una ética agonística ${ }^{1}$}

\section{The agonal ethic: A look into discursive relations between teacher and students from an agonistic ethic}

\author{
Óscar Felipe Roa Sánchez²
}

Para citar este artículo: Roa, O. F. (2016). El aula agonal: una mirada a las relaciones discursivas entre profesor y estudiantes desde una ética agonística. Enunciación, 21(2) 256-270.

Recibido: 29-abril-2016 / Aprobado: 28-octubre-2016

\section{Resumen}

El siguiente trabajo se propone aportar elementos conceptuales en torno a la reflexión de las interacciones discursivas que se tejen entre profesor $y$ estudiantes dentro del aula escolar. Elementos que nos acercan a la problemática de reconocer una dimensión ontológica de oposición o negatividad en las relaciones sociales en general, y en los procesos de enseñanza/aprendizaje en particular. Si bien desde los estudios contemporáneos del discurso y de la filosofía política se ha considerado lo agonístico por su potencia evaluadora, por cuanto lo agonal configura las relaciones de los participantes no como enemigos sino como adversarios, no se le ha dado suficiente reconocimiento para un análisis de las prácticas discursivas y la manera como se desenvuelven en el aula. En este estudio se tiene, en primer lugar, el modelo de análisis conversacional en el aula propuesto por Sinclair y Coulthard (1975) y se consideran algunos aportes del enfoque sociocomunicativo de Charaudeau (2012), para describir su entramado situacional. En segundo lugar, se trabaja el discurso pedagógico como dispositivo que transmite, no solo contenidos particulares según la disciplina, sino también como uno a partir del cual se establecen órdenes, relaciones e identidades sociales (Bernstein, 1994). Por último, la caracterización de una ética agonística como orientación filosófica y política para encarar de manera activa las prácticas de la vida escolar (Mouffe, 2006). De esta manera, los resultados se relacionan con el desarrollo discursivo de la oralidad en participantes que se enfrentan a la solución de conflictos dentro de un aula agonal.

Palabras clave: discurso, aula escolar, pedagogía, ética.

\begin{abstract}
The following work intends to provide conceptual elements around the reflection of the discursive interactions that are woven between teacherstudent within the classroom. Elements that brings us to identify the problem of recognizing an ontological dimension of opposition or negativity in social relations in a general way, and in teaching-learning processes in particular. While since contemporary studies of discourse
\end{abstract}

1 El siguiente documento, adscrito al semillero de investigación Hermeneia, de la Universidad Distrital Francisco José de Caldas, es un avance teórico sobre el proyecto investigativo titulado: "Los géneros discursivos en las interacciones cotidianas".

2 Estudiante investigador del semillero Hermeneia, de la Universidad Distrital Francisco José de Caldas, Bogotá (Colombia). Correo electrónico: felipe_roa22@hotmail.com 
and political philosophy has been considered the agonistic for its evaluative power, where the agonal sets up participants relations not as enemies but as adversaries, this aspect hasn't given sufficient recognition to the analysis of many discursive practices and the way as it develops inside a classroom scenery. In this study, we have in first place the Discursive Analysis model in the classroom from Sinclair and Coulthard (1978) and consider some contributions from a Socio-Communicative approach of Charadeau (2012), to describe situational elements from the classroom. In second place, we explain

\section{INTRODUCCIÓN}

Toda ciencia está en nosotros; la escuela, si no está basada en la pugnacidad, en la creación, perjudica.

Fernando González, Los negroides (1995).

Toda relación humana, en términos de lenguaje, es una interacción que se apoya en la mirada evaluadora -polémica- de los participantes que la constituyen. Es esta capacidad dinámica de construir tejidos sociales donde cada persona logra ser y conocer, donde es capaz de construir con el otro su mundo de la vida. Esto nos plantea la necesidad de una concepción del lenguaje orientada a la significación de la experiencia a través de múltiples códigos y formas de simbolización. De ahí la importancia de entender las interacciones comunicativas, en sus distintos escenarios, como el acto educativo por antonomasia en su valor de provocar la creación e interpretación de sentidos, sin duda, necesarios a la hora de encarar de manera activa y responsable los diferentes ámbitos de la vida.

En palabras de Calsamiglia y Tusón (1999), el lenguaje es "[...] la manera como -las personas- se ponen en funcionamiento para construir formas de comunicación y de representación del mundo (real o imaginario)" (p. 15) (resaltado por fuera del texto original). Un complejo sistema que se materializa
Pedagogical Discourse as a device that broadcast not only particular contents according to a discipline, but also as one of the aspects from which to stablish orders, relations and social identities (Bernstein, 1994). Finally, the characterization of an Agonistic Ethic as a political and philosophical orientation to confront in an active manner, scholar practices (Mouffe, 2006). This way, the results are related with the development of orality in situations that require the solution of problems inside classroom.

Keywords: discourse, classroom, pedagogy, ethic.

en gran medida a través de dos medios -el oral y el escrito- que dan lugar a dos modalidades de realización: la oralidad y la escritura. Además, de las múltiples funciones que desde los inicios de la sociedad ha tenido en la vida más privada o íntima, este ha ocupado también un lugar muy importante en la vida pública, institucional y religiosa. Especialmente en las realizaciones orales. Por eso es muy difícil pensar, por ejemplo, procesos de enseñanza y aprendizaje en la escuela sin la palabra dicha (Calsamiglia y Tusón, 1999).

En ese sentido, el interés por abordar prácticas comunicativas dentro del aula de clase, en relación con la complejidad intrínseca de procesos de comprensión y producción discursiva, su naturaleza, usos e intencionalidades, gira en torno al reconocimiento de la lengua como medio y objeto de aprendizaje. Es decir, la lengua considerada como actividad que existe en y por las prácticas lingüísticas de quienes la usan. Esta noción, así planteada, permite situar las prácticas orales en un plano interaccionista, por cuanto se desarrolla en el seno de esferas sociales (Bajtín, 1989) que implican intercambio, reconocimiento de sí y del otro, negociación, contextualización, movimiento y saber expresivo.

También tiene que ver con las experiencias de los investigadores de este proyecto que, como estudiantes de pregrado de Licenciatura en Humanidades y Lengua Castellana de la Universidad 
Distrital Francisco José de Caldas, se han desarrollado en torno a una preocupación por el estado de subordinación o subvaloración de la enseñanza de la oralidad dentro del aula escolar. Y cuyos efectos se discuten aquí desde un ideal de escuela contemporánea que se preocupa por formar ciudadanos desde el dominio discursivo oral como factor importante de inclusión en los diversos ámbitos de la vida social.

En general, la pregunta por el papel del discurso oral en el desarrollo de competencias comunicativas -agonísticas ${ }^{3}$ - dentro del escenario escolar, será el punto de partida de esta reflexión. Particularmente, en las implicaciones éticas de la actividad de hablar en los procesos de enseñanza y aprendizaje, cuyo abordaje exige una dimensión interdisciplinar de carácter discursivo, filosófico y político que posibilitan su valoración y comprensión en cualquier entorno de la vida cotidiana.

\section{AULA ESCOLAR: ACTO, MOVIMIENTO, INTERCAMBIO ${ }^{4}$}

Para empezar, diremos que una de las esferas sociales (Bajtín, 1989) que contiene procesos discursivos orales y escritos, y que además culturalmente se ha constituido como interfaz entre el individuo y la sociedad-conocimiento, es la escuela. Hablar de la escuela significa reconocer el valor social que cumple la educación en función de unos vínculos interpersonales que produce y reproduce. Aristóteles (1988), por ejemplo, planteaba que el objetivo principal para la educación era preparar a los jóvenes para asumir a largo plazo posiciones de liderazgo en las tareas del Estado; que la educación garantizara al ciudadano una vida políticamente plena en el ámbito de su comunidad.

3 Lo agonístico es aquí tratado a partir de los trabajos desarrollados por la experta en teoría política Chantal Mouffe: En torno a lo Político (2007); Agonística. Pensar el mundo políticamente (2014).

4 Los apartados que a continuación siguen aparecen en el libro Los géneros discursivos en las interacciones cotidianas (2016) realizados por el autor de este mismo artículo. Dicho libro constituye el trabajo de investigación logrado por el grupo Hermeneia. En el presente documento, estos apartados han sido sometidos a algunas modificaciones.
Es esta perspectiva la que considera la oralidad, anclada a un escenario educativo, un punto inicial en la reflexión sobre las maneras como los participantes (profesor y estudiantes) se constituyen en seres sociales, seres políticos, a partir de la configuración de identidades que dan sentido a la esfera escolar. Esto es lo que Hymes (1996), desde un enfoque antropológico, plantea como competencia comunicativa para referirse al uso del lenguaje en actos de comunicación particulares, concretos e históricamente situados, y que en términos de una comunicación efectiva, esta es, el desarrollo interaccional y la consecución de acuerdos, exige una competencia diferencial donde las prácticas sociales se configuran en el reconocimiento del entorno contextual y las necesidades lingüísticas que este despierta. La esfera escolar, en ese sentido, afecta no solo el conocimiento del código lingüístico, sino también el conocimiento social y cultural tanto del profesor como de los estudiantes (Hymes, 1996, p. 15).

Así, estas prácticas desarrolladas en la escuela, particularmente en el salón de clases, aparecen en un formato desde el cual se puede estudiar sus reglas de producción, a la vez que da cuenta de las identidades generadas en el ejercicio de construcción y reproducción de significados. Configuraciones que resultan comprensibles gracias a los estudios desarrollados sobre el contexto en su dimensión cognitivo-experiencial del que depende en gran medida el sentido y el valor de las palabras dichas ${ }^{5}$. Y que desde un análisis del discurso (AD), nos permite abordar con mayor detalle todo el entramado de secuencias y formas de enunciación que se ponen en juego cuando aparece un

5 Frente al estudio del contexto, son muchas las corrientes disciplinares que lo han tomado en función del análisis de la comunicación verbal como interacción social y el papel que cumple en los procesos de codificación y decodificación de la información. Como no es el interés de este documento hacer un análisis detaIlado sobre este concepto tan problemático, baste decir que aquí haremos referencia al contexto desde una perspectiva sociocognitiva que lo ubica no como un elemento superpuesto ajeno al proceso comunicativo sino como esquema cognitivo y experiencial que determina sistemáticamente la adecuación de las expresiones (Van Dijk, 2002). 
discurso, como el pedagógico, en clase. Por eso, pensar el aula en contexto implica: "[...] la manera como los participantes y sus acciones comunicativas (verbales y extraverbales), inmersas en características del entorno físico, resultan relevantes para el evento y los efectos -cognitivos- que produce esta acción comunicativa" (Calsamiglia y Tusón, 2012, p. 106).

Dicho esto, revisemos rápidamente el cuadro que Kerbrat-Orecchioni (1998, modificado por ViIlalta, 2009) ha elaborado para explicitar los niveles de desarrollo de una conversación (figura 1).

En la figura $1^{6}$, el autor nos presenta algunas categorías que se consideran a la hora de describir un proceso comunicativo. En primera medida, determina dos grandes unidades de análisis: contexto socioespacial y proceso de participación. Una relación importante a la hora de distinguir entre las situaciones reales de enunciación en toda su multiplicidad, los rasgos que son cultural y lingüísticamente pertinentes en la producción e interpretación de enunciados. Un ejemplo de ello son las maneras en que profesor y estudiantes forman marcos situacionales para el acto comunicativo dentro del aula de clase, y que activan unas expectativas en forma de guion "[...] que nos indican cómo actuar y cómo esperar que actúen los demás" (Calsamiglia y Tusón, 2012, p. 110).

Siguiendo con el esquema, en esas dos grandes unidades se desprenden otras unidades que a su vez contienen categorías que soportan la interpretación del entramado comunicativo (oral) como resultado de una serie de condicionamientos cognitivos, textuales y contextuales. Esto último clave en la actividad conversacional, pues nos permite reinterpretar en la marcha los papeles o roles de los participantes (sin perder rasgos como el de estatus) en su relación de negociadores tanto en los propósitos iniciales como en sus posibles desenlaces. Esto quiere decir que una formación del lenguaje no se reduce a poder preguntar y responder para garantizar el éxito de la comunicación, sino la conciencia de su uso como discurso en interacción con unas condiciones socioculturales y lingüísticas que controlan el evento comunicativo y que regulan las relaciones entre los participantes.

En definitiva, estos elementos del esquema aparecen en doble vía: por un lado, sirven de ruta metodológica a la hora de investigar y describir la conversación anclada a un escenario particular. Y por el otro, son estos mismos elementos los que indican el grado de desarrollo de competencias

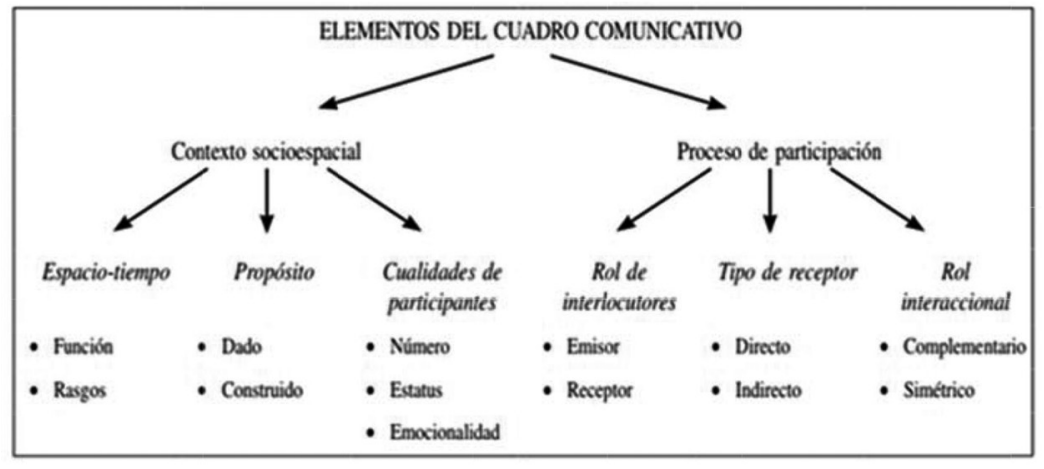

Figura 1. Cuadro comunicativo

Fuente: adaptado de Kerbrat-Orecchioni (1998, citado en Villalta, 2009).

6 Por lo planteado al inicio del documento, solo interesa en este punto hacer una descripción muy general del esquema que justifica su inclusión en esta reflexión. Para mayor información sobre el tema, revisar el documento de Marco Villalta (2009). 
comunicativas de los interlocutores, es decir, las formas de participación según las situaciones comunicativas (Villalta, 2009).

El aula de clase, en este sentido, se considera fundamentalmente como escenario comunicativo espacio-temporal donde convergen unos participantes principales del proceso educativo (profesor y estudiantes); cada uno con unos propósitos u objetivos que se especifican en gran medida por los lugares que allí ocupan: según el protagonismo discursivo (Hernández, G., 2006). Observar quién toma la palabra, cuántas veces, de qué manera y cuánto tiempo ocupa a lo largo de la interacción, aporta información importante sobre los papeles comunicativos que adopta cada participante y las relaciones de poder, dominación, de solidaridad o distancia que se puedan establecer (Calsamiglia y Tusón, 1999).

Podemos decir de todo esto que un primera aproximación sobre la conversación dentro del aula aparece como estructura comunicativa anclada a un escenario de práctica social (educación), donde los roles interaccionales entre profesor y estudiantes pre-existen en tanto se establecen unas posiciones en relación al discurso y sus objetivos ${ }^{7}$. Es decir, una condición que culturalmente se establece para "regular los intercambios comunicativos, y como consecuencia de ello, instaurar regularidades discursivas" (Charaudeau, 2012, p. 29).

Sin embargo, los elementos preexistentes a la producción discursiva oral no se mantienen estables en el tiempo que dura la interacción. Lo común es que el mismo proceso dialogal transforme la situación inicial o preestablecida. Esto obedece a la naturaleza viva de las prácticas a la que nos referimos, a situaciones auténticas de lo cotidiano que a pesar de estar atravesadas por dispositivos simbólicos que la definen como escenario educativo, escapa a lo emergente, a la palabra espontánea de los discursos orales que aparecen y se

7 Para mayor profundización sobre este aspecto del análisis discursivo, revisar la distinción entre escenario general y escenario específico, establecida por Charaudeau (2012). contraponen a las aproximaciones implantadas desde la teoría. En suma, hablar sobre el aula de clase es acercarse también a la vida humana en sus infinitas posibilidades.

Ahora bien, un aporte conceptual a la interacción dentro del aula de clase la encontramos en el modelo propuesto por Sinclair y Coulthard (1975, citados por Álvarez, 2008), donde establecen unidades básicas del intercambio y que consideramos son importantes para el ejercicio de análisis discursivo, en el sentido de poder despejar los diferentes niveles de la comunicación para organizar los momentos que la práctica comunicativa va adoptando según las disposiciones e intereses que los participantes le imprimen.

Estos autores proponen tres series de unidades del proceso comunicativo jerárquicamente ordenadas: 1) unidad de la gramática, 2) unidad de la organización no lingüística y 3) la unidad del discurso. A partir de estas, plantean los conceptos de acto, movimiento, intercambio, secuencia e interacción como niveles de producción en orden ascendente para configurar la unidad del discurso como eje que articula tanto lo lingüístico como lo no lingüístico (p. 15).

La interacción es el nivel mayor y queda delimitado por los procesos de apertura y cierre. La secuencia vendría delimitada por aspectos temáticos o por la alteración del rol de los interlocutores. El turno o movimiento es todo lo que dice un hablante antes de que comience otro. El intercambio está compuesto por dos o más turnos; es la unidad dialogal mínima. Dentro de un turno puede haber uno o más actos. El acto representa la unidad menor del nivel discursivo, se encuentra asociado con el traspaso de información de un punto a otro (elicitación), y se le reconoce a partir de las características de la situación y de elementos previos o posteriores al discurso. Ahora, "dichos actos constituyen movimientos que pueden ser de límite o de enseñanza" (Pilleux, M, 1994, p.11)

Este modelo se interesa por las propiedades funcionales de la comunicación, es decir, actos comunicativos que aparecen con intencionalidades 
previas (de uno de sus participantes) que moldean las disposiciones de los interlocutores y marcan, según dichos intereses, un movimiento particular en la interacción educativa. De ahí que su gran aporte tenga que ver con la demarcación de prácticas regulares que se desarrollan dentro del aula donde una acción, por ejemplo, de respuestas o excusas sigue a preguntas o peticiones; las aceptaciones o rechazos a ofrecimientos, entre otros.

En efecto, los procesos de enseñanza/aprendizaje dentro del salón de clase se construyen a partir de

[...] movimientos que consisten en una iniciación o turno que en este caso se representa por el que toma el profesor (I), seguido de una respuesta del estudiante $(R)$, seguida de una reacción del profesor a esta respuesta. Esta reacción última incluye, fundamentalmente una valoración o evaluación (E) (Álvarez, 2008, p. 16).

En ese sentido, es pertinente examinar con mayor detenimiento algunos elementos de este modelo (figura 2). Tenemos en los intercambios de límite el enmarcamiento que el profesor utiliza para dar fin a un intercambio e inicio a otro. En relación con esto, Calsamiglia y Tusón (1999) señalan que en el lenguaje existen marcadores discursivos, es decir, piezas lingüísticas (por ejemplo: bueno, consideremos ahora, dicho esto, con el fin de, etc.) que contribuyen a la organización global del texto en el tiempo y el espacio. Ahora, es importante señalar que estos marcadores de enmarcamiento no se orientan a los contenidos del discurso, sino ordenan el desarrollo del mismo. También hay marcadores no verbales o kinésicos que organizan la interacción, así que las realizaciones más típicas por parte del profesor serían: cruzarse de brazos, dirigir las acciones de participación con la mano, entre otras (Calsamiglia y Tusón, 1999, p. 48).

Con el enfocamiento el profesor anuncia lo que va a ocurrir o lo que ha ocurrido. Esta clase de turno tiene que ver más con el momento de retroalimentación o instalación de los participantes a la clase. Tenemos, por ejemplo, marcadores discursivos de los que hace uso el docente para iniciar su intervención: "antes de empezar", "tomen asiento, jóvenes", "el día de hoy", "para terminar", etc. De igual modo, hay marcadores kinésicos que refuerzan el objetivo del profesor por alcanzar la sufriente atención del grupo y direccionar las acciones futuras o que se han planteado con anterioridad para el desarrollo de la clase.

Ahora, en los intercambios de enseñanza la apertura consiste en hacer que otros participen en la toma de la palabra; por lo general se produce luego de que el profesor ha seleccionado al estudiante que debe responder. Se representa

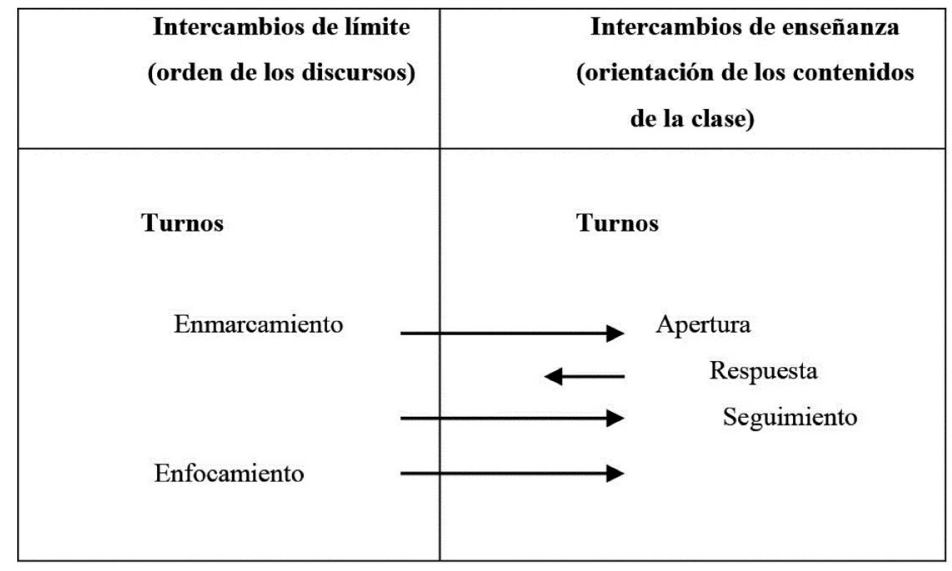

Figura 2. Intercambios comunicativos dentro del aula de clase

Fuente: adaptado de Pilleux. 
generalmente como apertura directiva y marca el inicio de los contenidos discursivos, es decir, la conversación propiamente dicha entre profesor y estudiantes.

Las respuestas a esta apertura se desarrollan según el movimiento discursivo que propone el profesor (declarativo, interrogativo, imperativo $)^{8}$. Y el seguimiento opera como una respuesta por parte de este que generalmente se presenta como evaluación o interrogatorio. Es en este punto del modelo, donde cobra mayor interés para este trabajo el intercambio de apertura ya que como escenario comunicativo que supone el aula de clase, nos permite examinar las maneras en que el discurso pedagógico, es decir, con el que se identifica al profesor, permite (o no) el surgimiento de otros discursos en el proceso de enseñanza/aprendizaje.

Para explicar mejor este punto, tomemos tres formas comunicativas recurrentes en la clase y que son culturalmente compartidas por el profesor y estudiantes (Pilleux, 1994): declarativo, interrogativo, imperativo como categorías gramaticales, representantes típicos de declaración, pregunta, orden como categorías situacionales, correspondientes a las categorías del discurso informativo, elicitación, directiva. Un proceso gradual que determina actos de habla, en eventos de habla, ubicados en situaciones de habla (Hymes, 1996).

Existen, empero, momentos que este modelo en su rigurosidad descriptiva no ha profundizado y que para los propósitos de este trabajo resultan importantes pues nos brinda pistas de actos que pueden explicar mejor las estrategias pedagógicas que acercan (o no) al estudiante a la posesión de la palabra como ejercicio del pensamiento que lo desprende de verdades definitivas representadas en el profesor, por tanto, un acto liberador.

8 Los movimientos de respuesta, por parte de los estudiantes, se complementan con las respectivas aperturas, por parte del profesor, y están predeterminadas por ellas: a) si la apertura es informativa, la respuesta generalmente es un acuse de recibido; b) si la apertura es una directiva, se espera una reacción; c) si la apertura es en forma de elicitación, se responde con una réplica, seguida a veces de un comentario (Pilleux, 1994).
En ese sentido, para una segunda aproximación del aula escolar es necesario considerar las variaciones secuenciales de límite y de enseñanza según las particularidades de unos objetivos didácticos, el número de participantes, las diferencias culturales, entre otras, que determinan los usos del lenguaje. Pues como ocurre en muchos casos, una apertura directiva puede realizarse mediante un interrogativo: "¿Están escuchando?", que quiere decir "¡Escuchen!"; o declarativo: "Está muy oscuro aquí", para decir "encienda la luz".

El turno de respuesta, por ejemplo, en un momento del desarrollo de la clase que además puede incluirse en la secuencia de exposición o diálogo de plenario ${ }^{9}$, es en sí misma una acción de apertura (cuando el estudiante responde y agrega una pregunta) y en ese instante puede ser que los roles de los participantes sufran cambios en la posición de poder-hablar; o que un seguimiento no sea más que una apertura a otros temas que puedan relacionarse con el principal. Estas posibilidades de lo imprevisible aparecen en el intercambio oral como momento crítico al análisis en el aula, pues nos acerca, siempre difuso e incompleto, a la fuerza o debilidad del poder estructurante que tiene el que provoca los intercambios (generalmente el profesor).

Por eso encontramos en los intercambios de límite y enseñanza de la conversación dentro del aula escolar espacios potencialmente polémicos que pueden reorganizar los actos entre un turno y otro de los participantes que, como ya se ha señalado, son los indicadores de movimientos que determinan un tipo de interpretaciones.

En todo caso, "[...] el estudio de la conversación-dentro del aula- permite conocer el nivel de estructuración predefinida y manejo de la incertidumbre que caracteriza este escenario educativo" (Villalta, 2009, p. 228) (resaltado por fuera del

9 Una secuencia se puede definir como un bloque de actos que establecen una coherencia de las diversas intervenciones y que están vinculadas por un significado o eje temático. Coherencia que se logra por la acción cooperativa de los hablantes para construir dicho significado. Para mayor información sobre secuencias en la interacción dentro de la sala de clases, revisar Villalta (2009). 
texto original). Un manejo en el nivel de recursividad que aún no se ha resuelto por parte de los interlocutores y que forma parte del proceso de enseñanza/aprendizaje en la escuela.

Ahora bien, es en estos momentos inestables de la conversación, donde se vinculan los trabajos de una ética agonística como constructo teórico-práctico que justifica el conflicto que se pueda presentar y que es necesario para la construcción de conocimientos significativos. Tal enfoque afirma que la negatividad es constitutiva a las prácticas sociales, y lo que inicialmente se ha acordado como principios ético-políticos que organizan una asociación -la clase en este caso-, pronto entra en conflicto por la interpretación que se hacen de dichos principios (Mouffe, 2014).

De manera que los intercambios en la clase, más que asegurar prácticas para transmitir información, deben ser estrategias discursivas que posibiliten un intercambio polémico entre los participantes que se encaminan siempre a consensos conflictuales (Mouffe, 2014, p. 27). Lo que Villalta (2009) plantea como "un dirigirse siempre al logro de 'satisfacción interaccional'" (p. 233). Los resultados de las negociaciones entre los interlocutores; la aceptación o rechazo de la complementariedad de lo dicho por el otro que aporta a la discusión de un tema específico en clase. Este grado de satisfacción nos permite visualizar el nivel de intercambio (de ajuste o negociado) que propone el profesor para el desarrollo de su clase ${ }^{10}$.

Pero antes de avanzar en esta idea, es importante considerar la dimensión sociocultural que configura o condiciona el discurso pedagógico y que explica en gran medida el tipo de prácticas educativas dentro del aula.

\section{DISCURSO PEDAGÓGICO}

Luego de estas consideraciones generales sobre la conversación en uno de los escenarios sociales

10 Frente a las categorías de ajuste y negociado, véase el trabajo de Álvarez (2008). más importantes, es necesario rescatar algunos trabajos sobre el discurso pedagógico como forma de acción que tiene el docente para generar efectos en la clase.

Como ya se ha mencionado, la reflexión sobre la comunicación oral en la escuela parte de los estudios sobre el análisis del discurso (ED), por eso reconoce en el trabajo elaborado por Bajtín (1989) Estética de la creación literaria, una base teórica importante para acercarnos con mayor detalle a la naturaleza compleja que entraña el estudio de este fenómeno del lenguaje, que en este caso refiere a un formato de la vida social: el discurso pedagógico dentro del aula.

En primer lugar, diremos con Bajtín (1989) que el discurso es una categoría abstracta, un constructo representacional de ciertas formas del lenguaje que responden a actividades sociales y que se encuentran dentro de la cultura de una comunidad. Por tanto, no supone una realización simple del lenguaje; cada proceso discursivo se considera como el resultado de una red compleja de relaciones sociales. En segundo lugar, y en consecuencia, el discurso no puede reducirse a la intención libre de un sujeto que articula significados; su producción se encuentra atravesada por un orden cultural en el cual se identifica y ubica sus enunciados.

Puede, en este sentido, situarse una primera clasificación del discurso pedagógico dentro de los géneros secundarios. Esta clasificación, realizada por Bajtín (1989), obedece a tres criterios: a) las condiciones sociales -políticas-, temáticas y discursivas de la esfera social; b) el nivel de relación que tienen los interlocutores con el contexto: inmediato o mediato; y c) el nivel de complejidad en la elaboración discursiva de los interlocutores. Obsérvese que esta clasificación, en orden descendente, le imprime a muchos discursos (institucionales específicamente) funciones constitutivas y regulativas.

La teoría bajtiniana propone que el discursivo representa la expresión verbal (oral y escrito), relativamente estable, cuya unidad es el enunciado que estructura un tipo temático, un tipo composicional 
y un tipo estilístico; además, de una determinada situación discursiva, posición social y relación entre los participantes. De manera que la escuela, como esfera social, representa un modo parcial de enunciados a los que podemos denominar géneros discursivos (Bajtín, 1989, p. 245).

Frente a esto, Patrick Charaudeau (2012) afirma que todo interés de análisis de discurso obedece a los niveles situacional, discursivo y formal que constituye todo escenario comunicativo: "[...] resulta -un género- de un contrato comunicacional y de la estrategia individual del sujeto" (p. 25) (resaltado por fuera del texto original). No obstante, este autor señala que para pensar un genero discursivo no se debería partir por un nivel formal (gramatical, léxico o fraseológico), ni por uno discursivo (modos de organización discursiva, modos enunciativos, tematización), sino desde un nivel de situación comunicativa, que reúne a los niveles ya mencionados pero como elementos constituyentes de un escenario comunicativo complejo por sus particularidades. De ahí que se considere importante describir el escenario escolar como etapa primera en la caracterización del discurso pedagógico, y de ese modo conocer los principios sobre los cuales se fundan los mecanismos de elección discursiva que se ponen en marcha a la hora de producirse el discurso del profesor.

Un tercer momento en la aproximación del aula de clase tiene que ver con la situación específica de comunicación (SEC) que regula los intercambios lingüísticos y, en consecuencia, establece los términos de la situación global de la comunicación (SGC). Esto es, la materialización singular de los dispositivos conceptuales que definimos dentro del campo educativo, a saber, "las identidades de los participantes, la finalidad que se define en términos de objetivos discursivos y un ámbito temático que constituye su basamento semántico" (Charaudeau, 2012, p. 31).

Otro aporte bajtiniano importante es la idea de sobrepasar los límites de posturas lingüísticas tradicionales que priorizaban una función expresiva de un hablante y al objeto de su discurso como fuerza discursiva única, dejando al oyente como otro medio o acompañante de su actividad individualista. Se produce entonces un giro a dicho esquema y se reconoce al oyente como interlocutor válido que frente a un primer enunciado del enunciador toma una postura activa de respuesta: "[...] el oyente, al percibir el significado de un discurso asume una respuesta [...] pues toda comprensión de este, de un enunciado viviente, tiene un carácter de respuesta" (Bajtín, 1989, p. 254).

Esto último resulta fundamental para comprender la noción de sujeto discursivo y sus relaciones agonísticas como base de una perspectiva del conflicto dirigida al aula de clase. Cobra vital importancia en este punto la afirmación que Bajtín (1989) hace sobre el discurso: "[...] en la realidad, cualquier comunicado va dirigido a alguien, está provocado por algo, tiene alguna finalidad, es decir, viene a ser un eslabón real en la cadena de la comunicación discursiva, dentro de una esfera determinada de la realidad cotidiana del hombre" (Bajtín, 1989, p. 270). En ese sentido, toda producción discursiva por parte del profesor despliega una preocupación interaccional frente a lo que el otro participante piensa, escucha y enuncia.

En definitiva, si afirmamos que toda manifestación del lenguaje en un escenario particular se explica por ciertas normas de comportamiento culturalmente establecidas y que se legitiman, por cuanto son asumidas para poder actuar en dichos escenarios $^{11}$, nos enfrentamos sin duda a una ética discursiva que configura los pensamientos y exige la acción del ser humano para alcanzar cierto nivel de autonomía en relación con el otro. El compromiso que se adquiere con uno mismo en el instante de interactuar con otras realidades igual de válidas y con las mismas posibilidades de reconocimiento. De ahí la importancia de reconocer esta dimensión en el comportamiento social de las personas a la hora de analizar manifestaciones discursivas en los diferentes escenarios de la vida cotidiana.

11 Discurso pedagógico dentro del aula de clase. 
Ahora, esta valoración del profesor por su discurso dentro de una situación específica de comunicación (aula de clase) se encuentra articulada a unas relaciones de poder y control simbólico (Bernstein, 1994). "El poder está siempre presente en cada discurso, y, a su vez cada discurso es un mecanismo de poder" (Salazar, 2014, p. 46). Y ha sido precisamente el discurso pedagógico uno de los mecanismos de poder y de control simbólico más efectivos para regular relaciones sociales. De ahí que pueda decirse que el discurso pedagógico es un dispositivo constituyente, contextualizante y recontextualizante de sujetos y sus relaciones.

En los trabajos realizados por Guadalupe Álvarez (2008) sobre el análisis de los desacuerdos en el discurso del aula, señala algunos elementos importantes:

Definiremos, a la manera como lo entendía Bernstein, el discurso pedagógico a partir de dos subdiscursos: I) como la regla que inserta un discurso de competencia (habilidades de tipo disciplinar y comunicativo) y en II) un discurso de orden social, de modo que el último domina siempre al primero. Llamaremos discurso instruccional al que transmite competencias especializadas y su relación entre ellas y discurso regulativo al que crea el orden especializado, la relación (con el otro) y la identidad (p. 17).

Los términos instruccional y regulativo complementan la reflexión sobre las prácticas educativas que se desarrollan en el aula de clase y que dirige el profesor. Bernstein (1994) rescata estos conceptos para redefinir el discurso pedagógico no solo como el que transmite contenidos particulares desde las diferentes disciplinas, sino también como uno a partir del cual se establecen órdenes, relaciones e identidades sociales (Álvarez, 2008). En otras palabras, su dimensión cultural y política nos permite analizar cómo un discurso instructivo se inserta en uno regulador, y dónde la selección, secuenciación y criterios del conocimiento, inmersos en el discurso pedagógico, están condicionados por las reglas que crean orden, relaciones e identidades (Bernstein, 1994).

Se pretende, en esta perspectiva, examinar el funcionamiento de la transmisión/adquisición de conocimiento, prestando atención no tanto a los productos de ese proceso, sino a los principios que lo hacen posible. "Si sabemos cuáles son los principios que subyacen a la pedagogía, podremos realizar tanto descripciones como explicaciones acerca de cómo funciona el proceso de transmisión/adquisición" (Bersntein, 1994, p. 137).

El discurso pedagógico como dispositivo es la condición que posibilita en gran medida la producción, reproducción o transformación de la cultura según las formas de control de los significados que dicho discurso permite. Bernstein (1994), en suma, pretende hacer evidente la influencia de los principios dominantes de la sociedad reflejados en la producción y reproducción de discursos pedagógicos materializados en prácticas para mantener un control simbólico.

Si bien estas evidencias de las fuerzas ideológicas, bajo las que opera el discurso pedagógico y con esto a los sujetos que lo viven, son determinantes, consideramos que la potencia de transformación (o de transgresión del género) en los intercambios regulativos (de enseñanza) e instruccionales (de límite) que guarda este discurso, es el punto de partida para entender los retos de la práctica crítica y reflexiva que se espera de los profesores, pues en su ejercicio se forma una actitud permanente de comprensión, interpretación y reconstrucción de su actuar pedagógico. Por tanto, resulta necesario un nuevo pensamiento, propio de una ética agonística, que opere permanentemente sobre el aula, donde la distancia entre los actores es el punto de contacto, que por supuesto no es físico sino más bien una posibilidad simbólica para la negociación entre profesor/estudiantes, profesor/texto, estudiantes/ texto. Esta negociación se quiere ver a la luz del diálogo que se presenta dentro de un aula agonal, asociada a la motivación, a una orientación 
metódica de la enseñanza con valores de clasificación, enmarcamiento y enfocamiento ${ }^{12}$. En suma, a la posibilidad de avanzar a la acción.

\section{ÉTICA AGONÍSTICA}

Es en el contexto de esta posibilidad del antagonismo que se ha elaborado una reflexión sobre las relaciones comunicativas entre el profesor y estudiantes. La intención es ofrecer una redescripción de las practicas discursivas que se llevan a cabo dentro del aula escolar a partir de los trabajos de una ética (Mouffe, 2014), cuyo aporte ha sido una dimensión agonística para el estudio de dichos intercambios.

Se considera en un primer momento la palabra pólemos de origen griego, que traduce "combate, polémica". Heráclito (citado por Spengler, 2013), considerado como el filósofo del devenir, proclama el movimiento como la esencia en la creación de la realidad en el mundo. Un devenir que descansa en una ley universal que rige todos los procesos cósmicos y humanos: "los opuestos" (p. 143). La construcción de conocimiento, desde esta perspectiva, implica oposición y se manifiesta en términos de una diferencia constante entre contrarios que complementa una unidad. El pólemos, en ese sentido, constituye la condición que hace posible que las cosas sean. Ahora, desde el análisis del discurso, se propone recuperar esta idea del contraste para justificar los beneficios de una ética que reconoce el conflicto en la actualidad como un problema de la no comunicación.

Esta ley de la discordancia, el contraste y la oposición, no desconoce la necesidad de un nosotros como síntesis de las diferencias, crucial para cuestiones políticas que no sacrifica el correlato de un ellos. La identidad de las cosas, dice Sepengler

12 El problema fundamental es cómo describir las formas de la comunicación pedagógica dentro del aula de clase y cómo ponerlas en relación con procesos histórico-sociales. La clasificación y el enmarcamiento son una de estas formas y refieren a principios que regulan las prácticas comunicativas en la relaciones entre profesor y estudiantes. Los conceptos y teorías constituyen el domino del discurso pedagógico (Bersntein, 1994, p. 60).
(1977), es su mismo ser diferente y opuesto, su mismo diversificarse y oponerse a las otras (p. 145). Es decir, que en las relaciones educativas en el salón de clases se considera "que el otro -profesor frente a los estudiantes- no es visto como enemigo a ser destruido o modelado, sino como reconocido adversario cuyas ideas serán combatidas -discursivamente-. Y ese derecho de defenderlas nunca será puesto en cuestión" (Mouffe, 2006, p. 21) (resaltado por fuera del texto original).

Para evitar malentendidos, es importante resaltar el contraste entre adversario y enemigo establecido por Mouffe (2014) para concebir una forma de democracia sin omitir la oposición; al derecho de cada individuo a diferir, a pensar y a vivir distinto, en síntesis, al derecho a la diferencia. Esto significa pensar como propiedad inherente al discurso pedagógico relaciones agonísticas dentro del aula de clase, así como en el resto del entramado social. Lo importante aquí, según Mouffe (2014), es que "el combate no adopte la forma de un antagonismo, que representa el enfrentamiento entre amigo/ enemigo y que por lo general termina en violencia física, sino la forma de un agonismo que significa el enfrentamiento -discursivo- entre adversarios" ( $p$. 32) (resaltado por fuera del texto original), cuyo resultado serán negociaciones que contribuyan a la gran pregunta de fondo de todo proyecto político y pedagógico: ¿Cómo poder vivir juntos?

Si bien hemos mencionado algunos elementos constitutivos de la palabra pólemos; abordemos ahora la palabra agón como complemento a este pensamiento. Para la Grecia arcaica, el agón era la fiesta donde los competidores se enfrentaban por un premio. Representaba para los griegos la lucha, sobre todo en concursos como los Juegos Olímpicos (Caravelo, 2012, p. 43). La competencia manifestada en los distintos escenarios sociales. Entonces, agón aparece como plataforma o escenario de la praxis social que posibilita la excelencia en los hombres, es decir, fomenta el pólemos de los individuos.

Una mirada contemporánea sobre estos elementos filosóficos nos acerca a la configuración 
del aula agonal como escenario comunicativo que se desenvuelve en espacios de diálogo polémicos atravesado por mínimos de convivencia -ética agonística- que garantizan el cambio y permiten la socialización de conocimientos. En efecto, como la lucha no consiste en aniquilar al rival sino en mantener una constante discusión, debe haber ciertas leyes de convivencia que los adversarios reconozcan y no transgredan (Caravedo, 2012, p. 53). Hay que decir en este punto, que la idea de redescripción en los intercambios de la comunicación que procura la enseñanza/aprendizaje en la escuela, exige la implementación base de un mínimo ético que permita y favorezca una participación activa de todos los que allí habitan.

Normas que garantizan un pluralismo agonista (Mouffe, 2006) que, por un lado, resisten a las tentativas de desactivar la diferencia connatural existente en las relaciones humanas (lo político) y, por el otro, se pregunta por los límites de un agonismo en cualquier tipo de contienda con cierto espíritu unitario que vincula a los participantes entre sí; el espíritu de pertenencia a un mismo Estado que motiva el respeto por las reglas de batalla entre los participantes y enlaza a la multiplicidad que subyace la pluralidad de lo existente (la política).

Desde este punto de vista, la escuela se traduce en un escenario de combate donde las posturas de la vida y de la educación del profesor se presentan ante las posturas y necesidades que traen los estudiantes. La clase se transforma en arena discursiva que, siguiendo con el modelo de Sinclair y Coulthard (1975, citados por Álvarez, 2008) reconfigura los intercambios de enseñanza tradicionales que han limitado las prácticas discursivas en un proceso de transmisión/adquisición, y resiste así los niveles de control simbólico que reducen habilidades y potencialidades tanto del profesor como de los estudiantes.

Esto es, actos agonísticos que transitan entre lo individual y lo colectivo, entre mundos de la vida y la articulación social, donde lo primero generalmente ofrece resistencia a lo segundo. Este problema es lo que Mauricio García (1991), reconoce como el camino de una ética en Colombia como proyecto político, educativo, que permita transformar actos de violencia históricamente desarrollados por escenarios de participación críticos y formativos. Planteando un reto imprescindible para todo proyecto político: "[...] transformar el miedo y el silencio, como fuente de la ética, en solidaridad y responsabilidad frente a la tarea de pertenencia a una sociedad y un mundo común" (p. 315).

De esta forma, reaparece un elemento fundamental en esta reflexión ética del discurso en la educación y tiene que ver con el pensar en el otro como elemento integrador de un nosotros que se construye en escenarios conflictivos. Una relación entre contrarios cuyo fin no es la destrucción del otro sino la consecución de acuerdos como respuesta máxima a la participación activa de la diferencia. En ese sentido, la concepción de enseñanza también se modifica, pues como lo advertía Heráclito, el camino al entendimiento se mueve por el camino de la lucha, una lucha originaria, porque hace que los combatientes -participantes del aula- comiencen a surgir como tales; no en su simple arremeter contra algo ya existente, sino en su naturaleza de no ser lo otro de él mismo, por tanto la inevitable confrontación con todo lo que le rodea. Entonces lo que se aprende es el resultado de la interacción conflictiva entre mi mundo de la vida, que no es más que la experiencia en el pensar, y el sendero difuso de la pluralidad.

Para marcar un compás de movimiento y de lucha donde todos podamos ejercer el poder de participar con el otro dentro de una discordancia que preserva la vida, pues sin el otro soy carente de identidad. Por tanto serán "[...] los productores, los creadores, los poetas, pensadores y hombres del Estado -profesores también- los que sostendrán esta lucha" (Heidegger, 1994, p. 179) (resaltado por fuera del texto original).

Es por eso que la práctica pedagógica debe aparecer como proceso dinámico que busca de la verdad. Eso significa que aspira constantemente hacia ella y que tiene el deseo -mas no la seguridad- de poseerla. Su presentación dentro del aula tiene un 
carácter inminentemente agonístico que asegura el debate, pero sobre todo, se justifica en su potencia de enseñar a pensar.

\section{AULA AGONAL}

De esta manera, el aula agonal sostiene la necesidad de exponer a los integrantes de la institución educativa al debate continuo. De ahí los efectos positivos de un análisis del discurso pedagógico que inicie por el escenario comunicativo particular para interpretar los imaginarios sociales entorno a lo que debe ser la educación, la escuela y el aprendizaje del lenguaje en acción (oralidad en este caso).

Un caso es que a partir de las descripciones situacionales del aula escolar se reflexiona la participación discursiva del profesor en el proceso educativo con un saber propio; su estado real de lo teórico-práctico construido a través de la reflexión personal y dialogal sobre su propia práctica pedagógica, "específicamente en el proceso de convertirse en praxis, a partir de su propia experiencia en relación con el grupo de estudiantes y de los aportes de las otras prácticas y disciplinas que se interceptan con su quehacer" (Vasco, s.f., p. 2). De manera que es el uso del lenguaje dentro del aula de clase lo que nos permite establecer algunos parámetros conversacionales a partir de una ética discursiva que encuentra en el discurso pedagógico uno de sus mayores representantes.

Esto implica un nuevo objetivo en el aula: transformar el antagonismo potencial en agonismo (Mouffe, 2014); el despliegue de otro tipo de intercambios que permitan a todos los participantes encontrarse en su diferencia para consolidar valores sociales, y así alcanzar consensos de orden académico y de convivencia que aporten a las bases constitutivas de la democracia en sus valores ético-políticos.

En últimas, lo que se pretende es resaltar la importancia del debate que se pueda generar en la interacción docente/alumno bajo los presupuestos de una ética agonística. Un combate que aparece con nuevas reglas de juego comunicativo cuyo reconocimiento permitirá la intercomprensión y en consecuencia el desarrollo de actividades que representan las necesidades de los participantes. Y con esto, las prácticas dentro del aula transforman las maneras de decir, pues como se ha dicho anteriormente una de las características del contrato comunicativo que se ha venido dando en la escuela es la consecución mecánica de intercambios que se organizan por turnos, y estos a su vez, permiten una intervención mínima de los participantes en el evento, especialmente los estudiantes.

Ahora bien, esto no quiere decir que el problema se descargue por completo en las restricciones sistemáticas de un modelo social. Aquí se insiste en la reelaboración (consolidación de la institución educativa) del escenario educativo que garantice un consenso conflictual como principio pedagógico cuyas bases éticas no solo sean legítimas, sino necesarias; donde la enseñanza de y desde el lenguaje como discurso en interacción inicie por las situaciones de uso y el manejo de conocimientos necesarios para un desenvolvimiento adecuado ${ }^{13}$.

Es el caso del profesor que a pesar de sus restricciones institucionales es capaz de pensar no solamente en los intercambios de enseñanza sino también aquellos de aprendizaje, atribuyendo la importancia a los interlocutores de este contrato (identidad de estudiantes) desde un marco de tratamiento enunciativo en el cual la instancia mediadora se construye desde la neutralidad o distanciamiento. De manera que los turnos puedan dirigir -mas no reducir- la participación de los estudiantes dentro del aula de clase.

Como resultado, la construcción discursiva reúne los procesos de producción e interpretación dentro de una situación comunicativa específica. Y lo que suponía un cierre, a través del seguimiento, ahora es un turno que busca la apertura de su interlocutor. Lo que desde una perspectiva agonística significa recuperar lo político de las prácticas sociales; esto es, la

13 La reformulación en la finalidad y objetivos educativos que corresponden a intencionalidades psicosocio-discursivas que configuran la postura del sujeto hablante en el acto de lenguaje y que parte del intercambio lingüístico mismo. 
"dimensión antagónica que es inherente a todas la sociedades humanas" (Mouffe, 2014, p. 22) y que se legitiman en la medida que contribuyen a la construcción de homonia (tejer comunidad) en donde debemos pensarnos para estar con el otro (Montoya, 2014).

La consideración de estos aspectos: combate pólemos- y una síntesis ética son parte del fundamento de la propuesta ${ }^{14}$. Es evidente que se busca la interacción cara a cara (mirar, oír, sentirse observado, observar al otro, interpretarlo, etc.) y la posibilidad de sentir la palabra pronunciada que alimenta el recuerdo y la emoción. Esto último, muy importante en la reivindicación de una realidad afectiva de la persona en el momento de sus prácticas educativas. Los significados se construyen a partir de la interacción entre la realidad interior del sujeto y la realidad exterior en la que habita.

Igualmente, la idea de controvertir al alumno no es tarea fácil pues, como ya se ha mencionado, las diferentes fuerzas que intervienen en el proceso educativo han limitado el accionar del profesor y repercute directamente en las posibilidades de compartir con los estudiantes. Por eso, no es sorpresa que lo útil para la escuela es inútil para el estudiante. Si nos planteamos pensar el aula como un escenario de fuerzas de intercambio donde se desafía, se interpela al estudiante, este deja de ser un receptáculo de información y valores; ahora el estudiante asume una actitud de combate frente a lo que se le ofrece en clase.

Dicha transición es lo que va a generar un escenario agonístico, un escenario de aprendizaje colaborativo. Por eso el poder acá no se reduce a sus efectos negativos, donde el profesor se vuelve un verdugo y el saber el patíbulo al que se convoca al

14 De la imagen que el maestro construya en sus estudiantes, dependen muchas actitudes por parte de estos. Una de las habilidades que se le ha recalcado a un licenciado ha sido la fuerza discursiva. Esta fortaleza permite atraer a un auditorio para generar el debate-polemos-. Ahora bien, esta fuerza no -nto que pueda decir en un instante o el poder para opacar a los receptores que se sienten obligados a escuchar. Esta fuerza hace alusión al poder de mover a un público a la acción y al choque que esto puede causar. estudiante. Ese poder lo posee tanto profesor como estudiantes, e intercambiarlo es la función del primero para posibilitar la apertura de horizontes de los estudiantes y de él mismo.

Frente a esto, lo agonístico, como síntoma de la batalla que en el caso de la clase al establecerse relaciones sobre conocimientos, revela la naturaleza de los estudiantes de ir transformándose en cada momento, según las nuevas batallas que se les presenten en la vida social. Por lo que el discurso pedagógico institucionalizado que opera bajo criterios rígidos, de un deber ser como producto generalizante, anula definitivamente el agón.

\section{CONCLUSIONES}

Podemos decir que el aula agonal aparece como característica del escenario comunicativo (Charaudeau, 2012) que guía las interpretaciones sobre las relaciones entre múltiples variables que intervienen en el funcionamiento de la clase; por eso su importancia en la clasificación del escenario (salón de clase) a partir del grado de producción oral que se teje entre sus participantes ${ }^{15}$.

Este trabajo ha pretendido iniciar los primeros pasos de un camino hacia la reflexión del género discursivo pedagógico anclado a un escenario agonístico que apunte a futuras elaboraciones. Que pueda dilucidar a partir de un orden reflexivo, un orden práctico entre el puente enseñar/ hablar/aprender y que el uso moderado o desmedido de ese poder-hablar afecta la manera como se desarrollan las relaciones de aprendizaje dentro del aula. Así, se considera que al ejercer poder en la escuela, sus efectos son tanto negativos, como positivos, por cuanto producen sistemas de saber (Hernández, 2006, p. 10).

15 Por supuesto no desconocemos que es el lenguaje escrito el medio principal para la transmisión del conocimiento escolar, y que su prioridad no siempre es negativa o contradice propuestas sobre la oralidad. Sin duda, el aprendizaje del lenguaje requiere de estas dos realizaciones para garantizar un desarrollo adecuado en los estudiantes y que operan con mayor o menor grado de profundización según los objetivos didácticos planteados por cada profesor. 
Estudiar de esta manera el fenómeno educativo en la escuela nos da una visión más amplia de cómo funciona el aula en sus prácticas comunicativas. De igual modo, el valor del análisis discursivo como herramienta no solo descriptiva sino como primer paso para comprender el fenómeno escolar y, si es el caso, sus posibles transformaciones. Solo queda preguntarnos si acaso es posible pensar la relación profesor/alumno como la pensaba $\mathrm{Ci}$ cerón al referirse al buen orador: "Desde el origen de la humanidad, hablar-comunicarse- es situarse en un equilibrio de fuerzas" (Ciceron, 1997, p. 27). ¿Podemos llegar a ese equilibrio de fuerzas en el aula?

\section{RECONOCIMIENTOS}

Este trabajo se desarrolla dentro del marco de proyecto investigativo "Los géneros discursivos en las interacciones cotidianas", vinculado al semillero de investigación Hermeneia. Proyecto aprobado y financiado por el Centro de Investigación y Desarrollo Científico (CIDC) de la Universidad Distrital Francisco José de Caldas.

\section{REFERENCIAS BIBLIOGRÁFICAS}

Álvarez, G. (2008). Análisis del discurso del aula: desde el enfoque comunicativo-interactivo de la argumentación. Forma y Función, 21, 13-34.

Aristóteles (1988). La Política. España: Editorial Gredos S.A.

Bajtín, M. (1989). Estética de la creación literaria. México: Siglo XXI Editores.

Bernstein, B. (1994). La estructura del discurso pedagógico. Clases, códigos y control. Madrid: Morata.

Calsamiglia, H. y Tusón, A. (1999). Las cosas del decir. Manual del análisis del discurso. Barcelona: Editorial Ariel.

Caravedo, U. (2012). La buena Eris. Reflexiones en torno a la lógica agonal en Heráclito y Nietzsche. Instantes y Azares, XII(10), 37-54.

Ciaspucio, G. (2005). La noción de género en la Lingüística Sistémico Funcional y en la Lingüística Textual. Revista Signos, 57, 31-48.

Cicerón. (1997). El orador. Madrid: Alianza Editorial.
Charaudeau, P. (2012). Los géneros: una perspectiva sociocomunicativa. En: M. Shiro, P. Charaudeau y L. Granato (ed.). Los géneros discursivos desde múltiples perspectivas: teorías y análisis (pp. 19-43). Madrid: Ed. Iberoamericana.

García, M. (1995). El camino de la ética civil en Colombia. Balance y perspectivas. Theologica Xaveriana, 46, 283-316.

González, F. (1995). Los negroides. Ensayo sobre la Gran Colombia. Medellín: Editorial Universidad Pontificia Bolivariana.

Heidegger, M. (1994). Conferencias y artículos. Barcelona: Ed. Del Serbal.

Hernández, G. (2006). El ejercicio del poder del maestro en el aula universitaria. CPU-e, Revista de Investigación Educativa, 2, 1-17.

Hymes, D. (1996). Acerca de la competencia comunicativa. Forma y Función, 9, 13-37.

Montoya, M. (2014). El género discursivo epidíctico en el aula: el orgullo del maestro. Tesis doctoral. Bogotá: Universidad Distrital Francisco José de Caldas.

Mouffe, C. (2006). Democracia y pluralismo Agonístico. Derecho y Humanidades, 12, 17-27.

Mouffe, C. (2014). Agonística. Pensar el mundo políticamente. México: Fondo de Cultura Económica de Argentina, S.A.

Pilleux, M. (1994). Análisis del discurso. La interacción en la sala de clases (La "Escuela de Birmingham"). Documentos Lingüísticos y Literarios, 20, 9-15.

Salazar, I. (2014). La alegría de enseñar. Una pedagogía poética. Ibagué: Caza de Libros.

Sinclair, J. y Coulthard. R. (1975). Toward and analysis of discourse. The English used by teachers and pupils. Londres: Longman.

Spengler, O. (2013). Heraclito. Sevilla: Ediciones Espuela de Plata.

Van Dijk, T. (2002). Conocimiento, elaboración del discurso y educación. Revista Escibanía, 8, 5-22.

Vasco, C. (s.f.). Algunas reflexiones sobre la pedagogía y la didáctica. Recuperado de: http://ineduga.webcindario. com/pedagogiadidactica.pdf

Villalta, M. (2009). Análisis de la conversación. El estudio de la interacción didáctica en sala de clase. Estudios Pedagógicos, 35(1), 221-238.

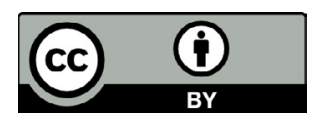

[ 270 ]

enunciación

ISSN 0122-6339 • ISSNe 2248-6798 • Vol 21, No 2 (julio-diciembre 2016). pp. 256-270. 


\section{Pedagogías de la lengua}

